\title{
Contribution of prior semantic knowledge to new episodic learning in amnesia
}

\author{
Irene P. Kan ${ }^{1,2}$, Michael P. Alexander ${ }^{3,4}$, and Mieke Verfaellie ${ }^{1}$ \\ 1 Memory Disorders Research Center, VA Boston Healthcare System and Boston University School of \\ Medicine, Boston, MA
}

2 Department of Psychology, Villanova University, Villanova, PA

3 Beth Israel Deaconess Medical Center, Boston, MA

4 Department of Neurology, Harvard Medical School, Boston, MA

\section{Abstract}

We evaluated whether prior semantic knowledge would enhance episodic learning in amnesia. Subjects studied prices that are either congruent or incongruent with prior price knowledge for grocery and household items and then performed a forced choice recognition test for the studied prices. Consistent with a previous report, healthy controls' performance was enhanced by price knowledge congruency; however, only a subset of amnesic patients experienced the same benefit. Whereas patients with relatively intact semantic systems, as measured by an anatomical measure (i.e., lesion involvement of anterior and lateral temporal lobes), experienced a significant congruency benefit, patients with compromised semantic systems did not experience a congruency benefit. Our findings suggest that when prior knowledge structures are intact, they can support acquisition of new episodic information by providing frameworks into which such information can be incorporated.

Decades of psychological and neuroscientific research have provided strong support for the distinction between episodic memory and semantic memory (Tulving, 1972, 1985). Whereas episodic memory refers to memory for personally experienced events (e.g., I dropped a hammer on my toe last night), semantic memory refers to memory for general world knowledge that is shared by others (e.g., Hammer is a tool used to drive in nails). Much of the existing research has focused on characterizing the cognitive and neural bases of each system, but relatively less is known about how the two systems interact. Nonetheless, data from healthy individuals and neuropsychological patients are beginning to provide important insight into the relationship between these systems. For example, neuroimaging studies of healthy individuals have provided evidence for the interdependence between semantic and episodic memory systems (e.g., Greve, van Rossum, \& Donaldson, 2007; Menon, Boyett-Anderson, Schatzberg, \& Reiss, 2002; Prince, Tsukiura, \& Cabeza, 2007; Rajah \& McIntosh, 2005; Wiggs, Weisberg, \& Martin, 1999). In addition, studies of semantic dementia highlight the role of episodic memory in semantic memory retrieval (Funnel, 1996; Graham, Simons, Pratt, Patterson, \& Hodges, 2000; Simons, Graham, Galton, Patterson, \& Hodges, 2001; Snowden, Griffiths, \& Neary, 1994, 1999). The current study examines whether (and how) prior semantic knowledge supports episodic learning in amnesia.

Address correspondence to: Irene P. Kan, Department of Psychology, Villanova University, 800 Lancaster Avenue, Villanova, PA 19085, Email: irene.kan@villanova.edu, Tel: 610-519-4720, Fax: 610-519-4269. 
Damage to the medial temporal lobes (MTL) results in dense amnesia, characterized by a profound impairment in episodic learning and relatively preserved semantic memory (Giovagnoli, Erbetta, \& Bugiani, 2001; Schmolck, Kensinger, Corkin, \& Squire, 2002). Although amnesic individuals are severely deficient in establishing new memories, anecdotal evidence suggests that some amnesics can be surprisingly good at learning new information that is relevant to their personal interests (e.g., an enthusiastic baseball fan recalling his favorite pitcher throwing a no-hitter the night before). How is such learning accomplished in the context of severe episodic encoding and retrieval impairments? There is ample evidence from studies with healthy individuals that episodic memory is enhanced when the to-be-remembered (TBR) information can be supported by pre-existing semantic (schematic) knowledge, suggesting that episodic encoding and retrieval processes may be more efficient when new information can be anchored to prior knowledge (Anderson \& Pichert, 1978; Bartlett, 1932; Brewer \& Treyens, 1981). We hypothesize that schematic activation may be a potential mechanism that can support episodic learning in amnesia.

We evaluated this mechanism by asking subjects to learn new information that is either congruent or incongruent with prior knowledge. We adapted our paradigm from a recent study that reported significantly better recall of prices of everyday items in healthy adults when price information was consistent with prior knowledge than when it was inconsistent (Castel, 2005). If anchoring new information to pre-existing knowledge enhances episodic encoding and retrieval (Minksy, 1975), we may expect to observe such a congruency benefit in memory for prices in amnesic subjects as well. A secondary question relates to patient variability in the ability to benefit from price congruency. We hypothesize that the capacity to benefit from semantic support may depend on the integrity of the pre-existing scaffolding: whereas an intact schematic network could support new learning, a weakened network could not exert such influence.

\section{Method}

\section{Participants}

Eight right-handed MTL-amnesics and 12 right-handed healthy controls participated in this study. The patient group had a mean age of 59.1 years $(\mathrm{SD}=12.7)$, an average of 15.1 years of education ( $\mathrm{SD}=2.5)$, and a mean verbal IQ of $107.5(\mathrm{SD}=20)$, as measured by the Wechsler Adult Intelligence Scale, Third Edition. In addition, all patients scored higher than $90 \%$ correct on the Pyramids and Palm Trees test, indicating that none of the patients had clinically significant impairments in semantic memory (Howard \& Patterson, 1992). The control group was matched to the patient group in terms of mean age $(\underline{M}=60.2$ years, $\mathrm{SD}=8.8)$, education $(\underline{\mathrm{M}}=14.8$ years, $\mathrm{SD}=1.6)$, and verbal IQ $(\underline{\mathrm{M}}=107, \mathrm{SD}=12)$, with all $\underline{\mathrm{t}} \mathrm{s}<1$. Table 1 summarizes the demographic and clinical neuropsychological data for the patients.

MRI/CT scans confirmed MTL pathology for all but two patients, as assessed by a board certified neurologist. One patient (P8) could not be scanned because he had a pace maker, and another patient's (P5) CT scan did not reveal any visible lesions. MTL pathology in both cases was inferred based on etiology. For the remaining patients with structural imaging data, two anoxic patients have damage restricted to the MTL, whereas one anoxic patient (P3) who also had a partial left temporal lobectomy and the three encephalitic patients have damage that extends to the anterior and lateral temporal lobes (see Figure 1).

\section{Design and Materials}

Stimulus selection-Eighty-four everyday items were selected. Color images of the products were obtained from the World Wide Web, and regular retail prices of these items were collected from online grocery stores and other online retailers. 
To ensure the prices we selected were realistic, we tested a group of six healthy adults $(\underline{\mathrm{M}}$ age $=60.2, \mathrm{SD}=8.4 ; \underline{\mathrm{M}}$ education $=14.8$ years, $\mathrm{SD}=3.1 ; \underline{\mathrm{M}}$ verbal $\mathrm{IQ}=107, \mathrm{SD}=15)$ on their price knowledge of this set of items in a two alternative forced-choice task. Each item was presented with a congruent target price (i.e., market value; $\$ 1.89$ for a dozen large eggs) and an incongruent foil price (i.e., $\$ 7.59$, approximately four times higher), and individuals were asked to select the price closest to the actual cost. They also rated how reasonable their selected price was on a 5 -point scale $(1=$ too low, $5=$ too high $)$. The experimental trials were randomly intermixed with 16 filler trials, on which the foil prices were approximately $20 \%$ of market prices (e.g., Cuisinart Toaster, \$5.99). Inclusion of filler items ensured that the correct response was not always the lower price of the pair.

Subjects performed well on the price knowledge task (mean accuracy $=96 \%$ ). We selected a final set of 64 items for the recognition memory task, on the basis of all healthy subjects having selected the target price and having rated the cost as reasonable ( $\underline{\mathrm{M}}$ rating across items $=3.11$, $\mathrm{SD}=0.43$ ). These selection criteria ensured that the prices were realistic reflections of healthy individuals' pre-existing price knowledge.

To verify that the selected items and prices were appropriate for use with amnesic patients, we also administered the price knowledge task to the patients. Although amnesic patients performed worse than controls, their performance on the forced-choice task was quite high ( $\underline{\mathrm{M}}$ accuracy $=89.9 \%, \mathrm{SD}=8.9 \%)$, indicating that their price knowledge is relatively intact. This task was administered at least two months prior to the memory task.

Materials-The 64 items were randomly divided into two sets of 32. Each subject encountered one set in the congruent condition and the other in the incongruent condition. Thus, items in the two conditions did not repeat for each subject, and congruency status of items in each set was counterbalanced across subjects. Each set of 32 was further divided into two lists of 16 to create two study-test blocks.

Each item was paired with a congruent price (i.e., market value) and an incongruent price (i.e., four times market value). Three foil prices to be presented at test were also generated for each item in each condition. Foils were created such that the span between the highest and lowest price options always stayed within $45 \%$ of the studied price. To ensure all price options appeared realistic, all prices ended with the digits " 0 ," "5" or "9" (e.g., Milk, gallon, "\$3.40," “\$3.85," "\$4.19," and " $\$ 4.90 ")$. Across trials, correct responses appeared in each of the four positions equally often.

\section{Procedure}

Each subject participated in two sessions, spaced at least one week apart, and items were blocked by price congruency across sessions. During each session, subjects participated in two study-test blocks of the same condition, separated by an unfilled 30-minutes delay to minimize potential interference. Condition order was counterbalanced across subjects. Prior to each block, subjects were told whether prices would be congruent or incongruent with market values. This was done to increase the likelihood of subjects engaging in the proper mental set for that block. Each session started with a practice block with four study and four test trials. These items were unique from the experimental set and were not included in data analyses.

Each study trial (verbal description, color photograph, and price) was presented for 10 seconds. Subjects were asked to read the item description and associated price aloud and to remember the item's price for a subsequent memory test. On each test trial, a verbal description and a photograph were presented with four prices, and subjects were given as much time as needed to select the studied price. A test block followed immediately after each study block. 
In accordance with the procedures of the Institutional Review Boards at the VA Boston Healthcare System and Boston University, all subjects provided informed consent prior to testing.

\section{Results}

The first set of analyses examines whether prior knowledge can support episodic learning. The second set of analyses evaluates whether the extent of semantic benefit may depend on the integrity of semantic memory.

For each subject, percent accuracy on the recognition test was calculated for each condition (see Table 2). Congruency benefit for each subject was defined as the difference in percent accuracy between the congruent and incongruent conditions.

\section{Semantic contribution to episodic memory}

A Wilcoxon Signed Ranks Test on recognition performance revealed a significant congruency main effect $(\underline{Z}=2.20, \mathrm{p}<0.05)$, with significantly better performance in the congruent condition $(\underline{\mathrm{M}}=58.8 \%, \mathrm{SD}=19.1 \%)$ than in the incongruent condition $(\underline{\mathrm{M}}=52.2 \%, \mathrm{SD}=$ $15.1 \%$ ). A group main effect (Mann-Whitney test, $\mathrm{Z}=3.71, \mathrm{p}<0.001$ ) confirmed that controls $(\underline{\mathrm{M}}=65.8 \%, \mathrm{SD}=11.5 \%)$ performed significantly better than patients $(\underline{\mathrm{M}}=39.9 \%, \mathrm{SD}=$ $6.8 \%$ ). Although the magnitude of congruency benefit differed numerically between groups, with controls showing a significant congruency benefit ( $\underline{\mathrm{M}}$ benefit $=8.9 \%, \underline{\mathrm{Z}}=1.96, \mathrm{p}=0.05$ ) and patients failing to show a congruency benefit ( $\underline{\mathrm{M}}$ benefit $=3.1 \%, \underline{\mathrm{Z}}<1)$, the interaction was not significant $(\underline{Z}=1.17, \underline{p}=0.24)$.

\section{Relationship between integrity of semantic memory and congruency benefit}

Neuropsychological findings have linked semantic memory storage and retrieval to the anterior and lateral temporal lobes (Hodges, 2003; Rogers, Lambon Ralph, Garrard, Bozeat, \& McClelland, 2004; Saffran \& Schwartz, 1994). Following from this idea, we indexed schematic scaffolding integrity with lesion profile and divided the seven patients with structural imaging data into two groups: those with damage that excludes anterior and lateral temporal lobes (i.e., damage restricted to the MTL, MTL-only group, $n=3)^{1}$ and those with damage that involves the anterior and lateral temporal regions in addition to the MTL (MTL-plus group, $n=4$; see Figure 1). The two groups of patients are matched on age, education, and VIQ (all p's > 0.85).

If integrity of the anterior and lateral temporal lobes indexes integrity of prior knowledge, MTL-only patients should perform substantially better on the price knowledge task than MTLplus patients, since the ability to correctly select the appropriate price for an item reflects preexisting knowledge. As predicted, MTL-only patients outperformed $(\underline{\mathrm{M}}=98.3 \%)$ MTL-plus patients $(\underline{\mathrm{M}}=85.5 \%)$ on the price knowledge task (Mann-Whitney, $\underline{\mathrm{Z}}=2.14, \underline{\mathrm{p}}<0.03$ ).

Furthermore, if schematic integrity impacts new learning, then MTL-only patients should experience a greater congruency benefit than MTL-plus patients. A Mann-Whitney test revealed a significantly larger congruency benefit in the MTL-only group $(\underline{M}=12.3 \%)$ than in the MTL-plus group $(\underline{\mathrm{M}}=-4.5 \%, \underline{\mathrm{Z}}=2.16, \underline{\mathrm{p}}<0.05)$. These data suggest that integrity of the anterior and lateral temporal lobes is critical for prior knowledge support in episodic memory (see Table 2).

Importantly, the group difference in congruency benefit cannot be attributed to baseline differences in episodic memory impairments. This is confirmed by the observation that patients

\footnotetext{
${ }^{1} \mathrm{P} 5$, the anoxic patient with no visible lesion on his CT scan, was classified into the MTL-only group.
} 
in the two groups performed similarly on two baseline episodic memory measures: on Auditory Delayed Memory, Wechsler Memory Scale-III (Wechsler, 1997b, MTL-only M = 62, SD = 15 , MTL-plus $\mathrm{M}=61, \mathrm{SD}=11, \mathrm{Z}<1)$ and on recognition memory in the incongruent condition (MTL-only $\underline{\mathrm{M}}=37.7 \%$, MTL-plus $\underline{\mathrm{M}}=39.0 \%, \underline{\mathrm{Z}}<1$ ).

\section{Discussion}

This study examined the contribution of prior knowledge to episodic learning in amnesia. After studying prices for everyday items, amnesic patients and controls performed a recognition test for the studied prices, which were either congruent or incongruent with prior knowledge. Whereas healthy controls experienced a price congruency advantage, only a subset of amnesics benefited from price congruency. We observed that the capacity to benefit from prior knowledge is dependent on the integrity of the long-term knowledge scaffolding, as defined by lesion extent: Whereas patients with intact prior knowledge experienced a congruency benefit, patients with weakened prior knowledge did not, even though these groups did not differ on two baseline measures of episodic learning. These observations are consistent with the idea that intact prior semantic knowledge supports new episodic learning.

One interpretation of the observed congruency benefit is that episodic encoding and retrieval are facilitated when the TBR information can be anchored to pre-existing knowledge. The "levels of processing" framework is one variant of this interpretation. By this view, episodic encoding is enhanced when new information is processed "deeply" (i.e., on a semantic level), which allows for active linking of incoming information with prior knowledge (Craik \& Lockhart, 1972). Another variant of the anchoring interpretation refers to the construct of "schemata"-abstract knowledge structures that are stored in semantic memory (Bartlett, 1932). When such structures are activated at encoding, they provide a framework to organize incoming information and also serve as bases for selective attention (Rumelhart, 1980; Schank $\&$ Abelson, 1977). This allows automatic anchoring of congruent incoming information to preexisting generic "slots" within the schematic scaffolding, which enhances encoding and subsequent retrieval (Anderson et al., 1976). On the other hand, when incoming information is inconsistent with the activated structures, encoding and retrieval processes become less efficient and more effortful (Arbuckle, Vanderleck, Harsany, \& Lapidus, 1990).

An alternative interpretation that might be offered for the observed congruency benefit is that rather than enhancing encoding and retrieval processes, schematic structures simply provide a useful guessing framework at test. By this view, rather than using the activated schema to organize new information, subjects simply use the activated schematic information to eliminate unlikely price options at test in the congruent condition. Such a strategy would not be beneficial in the incongruent condition, however, as all price options are equally unlikely.

To assess this possibility, we tested 12 naïve right-handed healthy controls $(\underline{\mathrm{M}}$ age $=58.5$ years, $\underline{\text { M education }}=15.3$ years) in only the recognition phase of the memory experiment. For each item in the congruent block, subjects were asked to select the price that was closest to market price for that item. For each item in the incongruent block, subjects were asked to imagine that they were in a foreign country where everything costs substantially more than in the United States and select the price that was closest to what they would expect to pay for that item in that country. If the observed congruency benefit is simply an artifact of increased guessing efficiency in the congruent condition, we should replicate the congruency benefit with this task. Each subject participated in both conditions, and trials were blocked by condition, with 32 trials in each. Chi-square tests revealed that subjects performed at chance under these instructions in both the congruent $\left(\underline{\mathrm{M}}=28.3 \%, \mathrm{SD}=6.2 \%, \chi^{2}<1\right)$ and the incongruent $(\underline{\mathrm{M}}=$ $\left.29.0 \%, \mathrm{SD}=8.4 \%, \chi^{2}<1\right)$ conditions. Furthermore, a direct comparison of the congruency benefit in the MTL-only group and the control group revealed a significant group difference 
(Mann-Whitney, $\underline{Z}=2.03, \underline{\mathrm{p}}<0.05)$. Taken together, these analyses argue against the notion of semantically guided guessing as the basis of the congruency benefit.

The idea that episodic learning is influenced by schematic knowledge can also be accommodated by neural network models of long-term memory (e.g., Alvarez \& Squire, 1994). For example, McClelland, McNaughton, and O'Reilly (1995) make a distinction between a fast hippocampally-mediated learning system and a slow neocortically-based learning system. Most relevant to the current study is the idea of "interleaved learning," which refers to the integration of new information from the fast system into pre-existing knowledge stored in the slow system. Thus, new learning can be enhanced if new information can be interleaved with pre-existing representations (for a similar proposal, see Murre, 1997). Our data and a recent report (Tse et al., 2007) of hippocampal-lesioned rats achieving one-trial learning when such learning can be supported by pre-existing knowledge extend this view, suggesting that rapid learning can be achieved to some extent despite hippocampal damage, so long as the relevant prior knowledge is intact.

Extending these observations, our data demonstrate that amnesic patients' episodic memory is enhanced when the TBR information can be anchored to intact prior knowledge. These results provide an important illustration of how intact schematic information can support episodic learning, even when episodic memory is impaired. It is important to note, however, that despite their severe episodic memory impairments, our patients do have some residual episodic memory functions. We speculate that in order to benefit from semantic contributions, in addition to intact semantic memory, minimal residual episodic memory functioning is required.

In this study, the impact of semantic knowledge on episodic memory was manifested as enhanced accuracy, but this may not always be the case. For instance, it has been reported that when amnesic patients confabulate, the content of their confabulations is often based on true personal history (Burgess \& McNeil, 1999; Schnider, 2003). In this case, established personal knowledge may interfere with the retrieval of episodic memories, leading to the intrusion of context-inappropriate information. Regardless of the nature of the influence (i.e., facilitation vs. interference), these observations are consistent with the idea that semantic memory impacts both episodic encoding and retrieval processes.

One question left unanswered by the present findings is whether MTL-only patients experience as large a semantic congruency benefit as do controls. A direct comparison of the magnitude of the congruency benefit in controls and in MTL-only patients is compromised by a potential scaling effect, as MTL-only patients and controls performed at different baseline levels. Also, because patients in the MTL-plus group all had bilateral lesions, it remains unknown whether a unilateral anterior/lateral temporal lesion in either the left or right hemisphere alone would abolish the congruency benefit. Given that pre-existing price knowledge is likely encoded and stored in verbal form and was accessed in the context of a verbal memory task, left hemisphere semantic structures may be essential for observing a semantic contribution in the current task. Future studies, evaluating patients with unilateral MTL-plus lesions on tasks that require access to both verbal and nonverbal semantics will be important to tease apart the conditions under which each hemisphere is important for semantic support. These limitations notwithstanding, the critical finding is that our data provide direct support for a semantic contribution to episodic memory in amnesia.

The generalizability of our findings also remains to be explored. For example, what is the efficacy of other types of schematic knowledge in supporting episodic learning? Will explicit instructions to exploit prior knowledge during episodic encoding yield additional benefit? Answers to these questions will provide further insights into how prior knowledge supports 
episodic learning and will advance our understanding of how long-term memory systems interact and support each other.

\section{Acknowledgements}

This research was supported by the NIH grants MH 57681 and MH71783 and the Office of Research and Development, Medical Research Service, Department of Veterans Affairs (awarded to MV) and the NIH grant HD 046442 (awarded to MPA). We thank Elizabeth Martin, Karen Fossum, and Sarah Davis for their assistance in data collection and Erin Sakai for her assistance in preparation of this manuscript.

\section{References}

Alvarez P, Squire LR. Memory consolidation and the medial temporal lobe: A simple network model. Proceedings of the National Academy of Sciences of the United States of America 1994;91:70417045. [PubMed: 8041742]

Anderson MC, Pichert JW. Recall of previously unrecallable information following a shift in perspective. Journal of Verbal Learning \& Verbal Behavior 1978;17:1-12.

Anderson MC, Pichert JW, Goetz ET, Schallert DL, Stevens KV, Trollip SR. Instantiation of general terms. Journal of Verbal Learning \& Verbal Behavior 1976;15:667-679.

Arbuckle TY, Vanderleck VF, Harsany M, Lapidus S. Adult age differences in memory in relation to availability and accessibility of knowledge-based schemas. Journal of Experimental Psychology: Learning, Memory, and Cognition 1990;16:305-315.

Bartlett, FC. Remembering. Cambridge: Cambridge University Press; 1932.

Brewer WF, Treyens JC. Role of schemata in memory for places. Cognitive Psychology 1981;13:207230.

Burgess PW, McNeil JE. Content-specific confabulation. Cortex 1999;35:163-182. [PubMed: 10369091]

Castel AD. Memory for grocery prices in younger and older adults: The role of schematic support. Psychology \& Aging 2005;20:718-721. [PubMed: 16420146]

Craik FIM, Lockhart RS. Levels of processing: A framework for memory research. Journal of Verbal Learning and Verbal Behavior 1972;11:671-684.

Funnel, E. Semantic dementia: A role for goals and scripts in the memory for object concepts in everyday situations. Paper presented at the British Neuropsychological Society Annual General Meeting; 1996.

Giovagnoli AR, Erbetta A, Bugiani O. Preserved semantic access in global amnesia and hippocampal damage. Cognitive Neuropsychology 2001;15:508-515.

Graham KS, Simons JS, Pratt KH, Patterson K, Hodges JR. Insights from semantic dementia on the relationship between episodic and semantic memory. Neuropsychologia 2000;38:313-324. [PubMed: 10678697]

Greve A, van Rossum MCW, Donaldson DI. Investigating the functional interaction between semantic and episodic memory: Convergent behavioral and electrophysiological evidence for the role of familiarity. Neuroimage 2007;34:801-814. [PubMed: 17112741]

Hodges, JR. Semantic dementia: A disorder of semantic memory. In: D’Esposito, M., editor. Neurological foundations of cognitive neuroscience. Cambridge, MA: MIT Press; 2003. p. 67-88.

Howard, D.; Patterson, K. The pyramids and palm trees test: A test of semantic access from words and pictures. Bury St Edmunds: Thames Valley Test Company; 1992.

McClelland JL, McNaughton BL, O'Reilly RC. Why there are complementary learning systems in the hippocampus and neocortex insights from the successes and failures of connectionist models of learning and memory. Psychological Review 1995;102:419-457. [PubMed: 7624455]

Menon V, Boyett-Anderson JM, Schatzberg AF, Reiss AL. Relating semantic and episodic memory systems. Cognitive Brain Research 2002;13:261-265. [PubMed: 11958970]

Minksy, M. A framework for representing knowledge. In: Winston, PH., editor. The Psychology of Computer Vision. New York: McGraw-Hill; 1975. p. 211-277.

Murre JMJ. Implicit and explicit memory in amnesia: Some explanations and predictions by the Trace Link model. Memory 1997;5:213-232. [PubMed: 9156099] 
Prince SE, Tsukiura T, Cabeza R. Distinguishing the neural correlates of episodic memory encoding and semantic memory retrieval. Psychological Science 2007;18:144-151. [PubMed: 17425535]

Rajah MN, McIntosh AR. Overlap in the functional neural systems involved in semantic and episodic memory retrieval. Journal of Cognitive Neuroscience 2005;17:470-482. [PubMed: 15814006]

Rogers TT, Lambon Ralph MA, Garrard P, Bozeat S, McClelland JL. The structure and deterioration of semantic memory: A neuropsychological and computational investigation. Psychological Review 2004;111:205-235. [PubMed: 14756594]

Rumelhart, DE. Schemata: The building blocks of cognition. In: Spiro, RJ.; Bruce, BC.; Brewer, WF., editors. Theoretical issues in reading comprehension: Perspectives from cognitive psychology, linguistics, artificial intelligence, and education. Hillsdale, NJ: Lawrence Erlbaum; 1980. p. 33-58.

Saffran, EM.; Schwartz, MF. Of cabbages and things: Semantic memory from a neuropsychological perspective - a tutorial review. In: Umilta, C.; Moscovitch, M., editors. Attention and Performance XV: Conscious and Nonconscious Information Processing. Cambridge, MA: MIT Press; 1994. p. 507-536.

Schank, R.; Abelson, R. Scripts, plans, goals and understanding. Hillsdale, NJ: Erlbaum; 1977.

Schmolck H, Kensinger EA, Corkin S, Squire LR. Semantic knowledge in patient H.M. and other patients with bilateral medial and lateral temporal lobe lesions. Hippocampus 2002;12:520-533. [PubMed: 12201637]

Schnider A. Spontaneous confabulation and the adaptation of thought to ongoing reality. Nature Reviews Neuroscience 2003;4:662-671.

Simons JS, Graham KS, Galton CJ, Patterson K, Hodges JR. Semantic knowledge and episodic memory for faces in semantic dementia. Neuropsychology 2001;15:101-114. [PubMed: 11216881]

Snowden J, Griffiths H, Neary D. Semantic dementia: autobiographical contribution to preservation of meaning. Cognitive Neuropsychology 1994;11:265-288.

Snowden J, Griffiths H, Neary D. The impact of autobiographical experience on meaning: Reply to Graham, Lambon Ralph and Hodges. Cognitive Neuropsychology 1999;16:673-687.

Tse D, Langston RF, Kakeyama M, Bethus I, Spooner PA, Wood ER, et al. Schemas and memory consolidation. Science 2007;316:76-82. [PubMed: 17412951]

Tulving, E. Episodic and semantic memory. In: Tulving, E.; Donaldson, W., editors. Organization of Memory. New York: Academic Press; 1972. p. 381-403.

Tulving E. How many memory systems are there? American Psychologist 1985;40:385-398.

Wechsler, S. Wechsler Adult Intelligence Scale, III. San Antonion, TX: Psychological Corporation; 1997a.

Wechsler, S. Wechsler Memory Scale, III. San Antonio, TX: Psychological Corporation; 1997b.

Wiggs CL, Weisberg J, Martin A. Neural correlates of semantic and episodic memory retrieval. Neuropsychologia 1999;37:103-118. [PubMed: 9920476] 
MTL-plus group
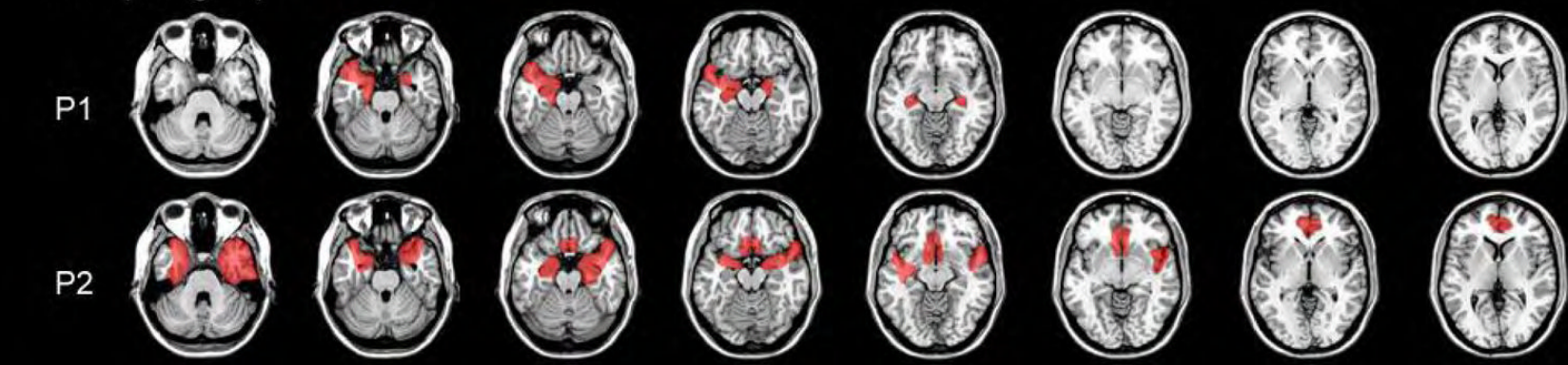

P3
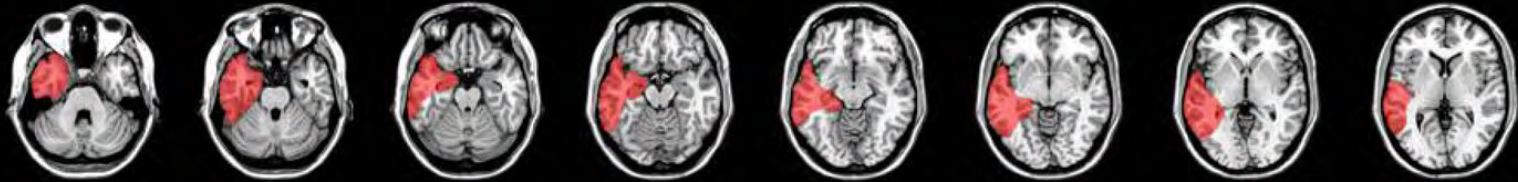

P4
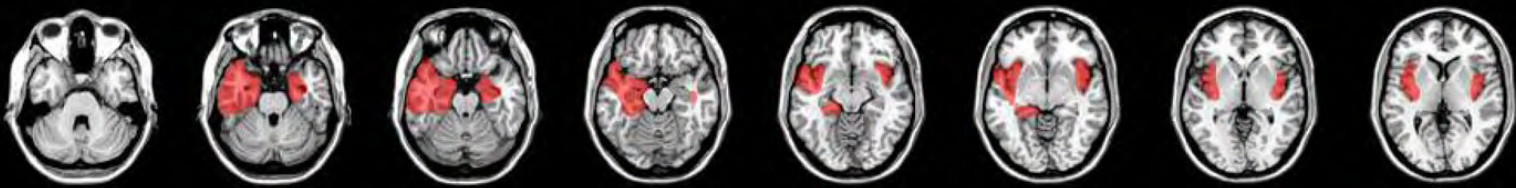

MTL-only group
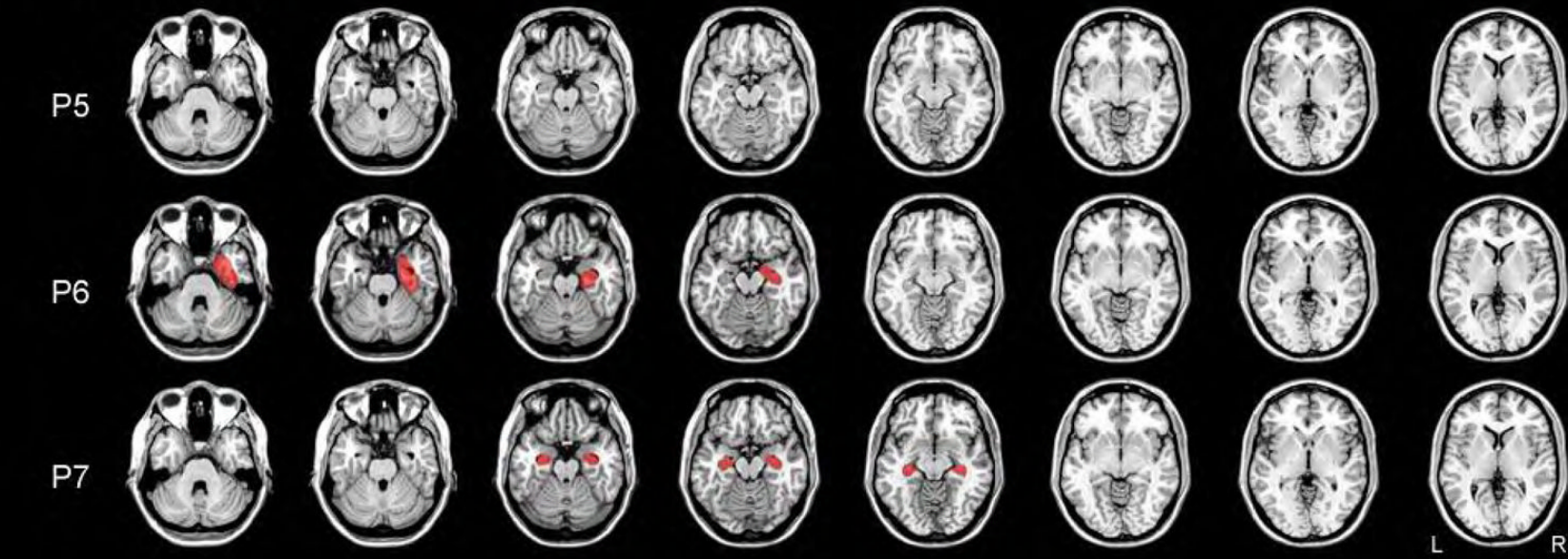

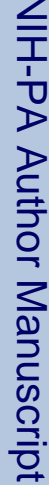

Figure 1.

Reconstruction of lesions based on CT and MRI scans. Patients were characterized into two groups: MTL-only (bottom) and MTL-plus (top). Whereas MTL-only patients have lesions restricted to the medial temporal lobes and spared the anterior and lateral temporal lobes, MTLplus patients have lesions that include the medial temporal lobes and the anterior and lateral temporal lobes. (P5 has no visible lesion on the CT scan). 


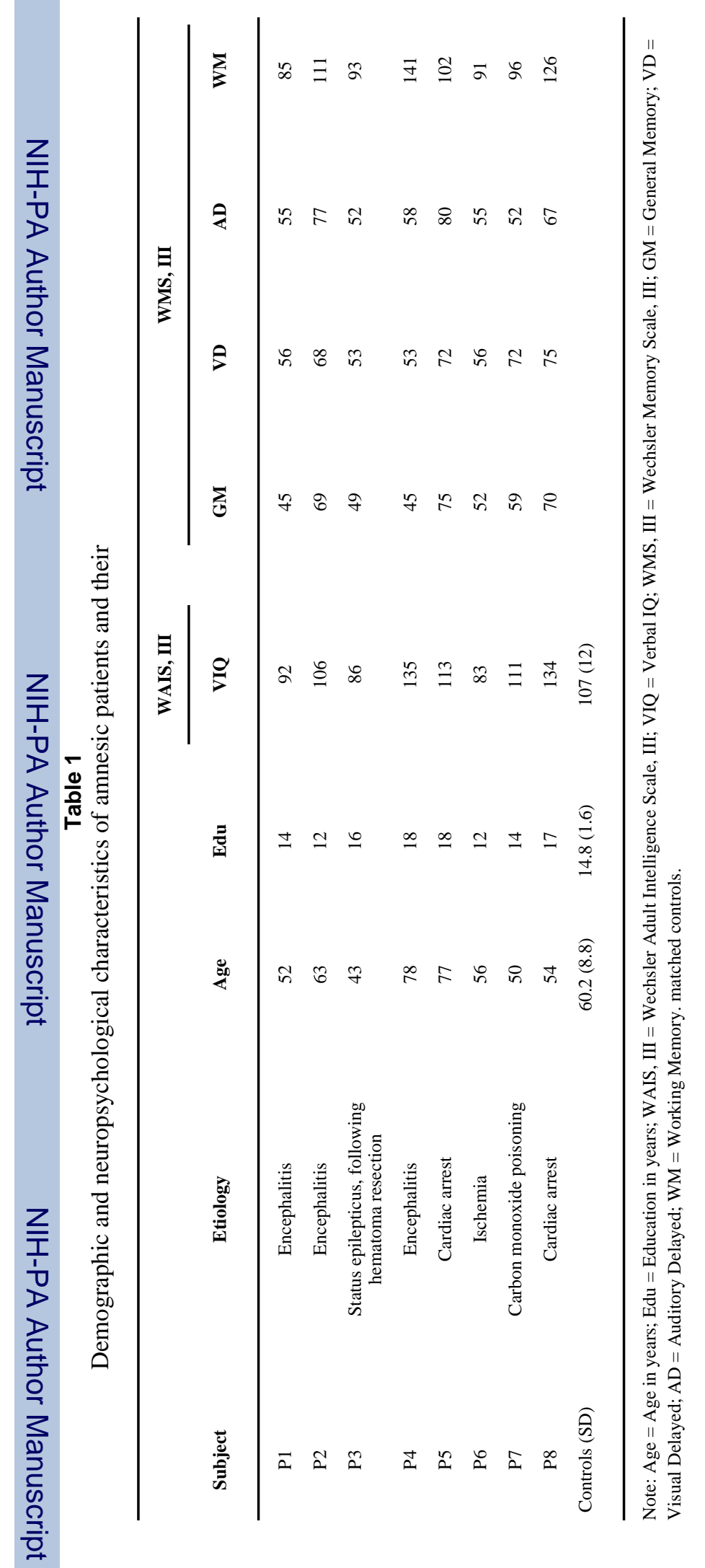

J Cogn Neurosci. Author manuscript; available in PMC 2009 May 1. 
Table 2

Individual patient's performance on recognition test and on prior knowledge task. Mean percent correct (and standard deviation) on recognition test and on prior knowledge task for each sub-group.

\begin{tabular}{ccccc}
\hline Subject & Group & Congruent & Incongruent & Prior knowledge \\
\hline P1 & MTL+ & $41 \%$ & $50 \%$ & $92 \%$ \\
P2 & MTL+ & $38 \%$ & $44 \%$ & $92 \%$ \\
P3 & MTL+ & $28 \%$ & $34 \%$ & $80 \%$ \\
P4 & MTL+ & $31 \%$ & $28 \%$ & $78 \%$ \\
P5 & MTL only & $53 \%$ & $41 \%$ & $100 \%$ \\
P6 & MTL only & $44 \%$ & $31 \%$ & $97 \%$ \\
P7 & MTL only & $53 \%$ & $31 \%$ & $98 \%$ \\
P8 & n/a (no imaging data) & $44 \%$ & $\mathbf{3 8 . 4 \% ( 7 . 2 \% )}$ & $94 \%$ \\
& All MTL patients & $\mathbf{4 1 . 5 \% ( 9 . 1 \% )}$ & $\mathbf{8 9 . 9 \% ( 8 . 9 \% )}$ \\
& MTL-plus & $\mathbf{3 4 . 5 \% ( 6 . 0 \% )}$ & $\mathbf{8 5 . 5 \% ( 3 . 8 \% )}$ \\
& MTL-only & $\mathbf{5 0 . 0 \% ( 5 . 2 \% )}$ & $\mathbf{3 9 . 0 \% ( 9 . 9 \% )}$ & $\mathbf{9 8 . 3 \% ( 1 . 5 \% )}$ \\
\hline
\end{tabular}

